\title{
EFECTO DE LA SEPARACIÓN MECÁNICA DEL MÚSCULO EN LA RETENCIÓN Y VIABILIDAD DE LAS LARVAS DE ANISAKIS DURANTE EL PROCESO DE OBTENCIÓN DE SURIMI
}

\section{EFFECT OF THE MECHANICAL SEPARATION OF MUSCLES ON THE RETENTION AND VIABILITY OF ANISAKIS LARVAE DURING THE PRODUCTION OF SURIMI}

\author{
Fabiola Olivares ${ }^{1}$, Cristina de las $\operatorname{Heras}^{2}$, María Teresa Solas $^{3}$ y Margarita Tejada ${ }^{4}$
}

\begin{abstract}
Resumen
El surimi, músculo de pescado picado y lavado, es generalmente utilizado como materia prima en la preparación de una variedad de productos de imitación cuyo consumo ha incrementado a lo largo de los años. Durante la elaboración del surimi o productos derivados, la congelación o calentamiento aplicados son suficientes para matar las larvas de Anisakis, sin embargo la presencia de larvas muertas aún puede causar síntomas de alergia en consumidores sensibilizados. El objetivo del estudio fue evaluar la presencia y viabilidad de larvas de A. simplex en el músculo tamizado y su retención en los tamices después de pasar el músculo infestado a través de tamices individuales (simulación de extrusión) o sucesivos (simulación de extrusión y refinado) con diferentes diámetros de orificio. Se utilizaron filetes de merluza (Merluccius merluccius) con infestación de larvas vivas de A. simplex (20 larvas/100 g de músculo) (infestación controlada) y una mezcla de musculaturas ventrales de merluza congelada-descongelada con una alta tasa de infestación natural (promedio 90 larvas/100 g de músculo) y se determinó la presencia de larvas en el músculo tamizado y su retención en los tamices. El rendimiento del músculo obtenido después del tamizado osciló entre 60 y $80 \%$ dependiendo del tamiz y materia prima utilizados. El porcentaje de larvas enteras (vivas o muertas) en el músculo tamizado disminuyó significativamente con el diámetro del orificio y cuando se utilizaron tamices sucesivos. El alto contenido de larvas rotas encontradas podría indicar una mayor dispersión de antígenos en el músculo tamizado.
\end{abstract}

Palabras clave: Anisakis simplex, separación mecánica, surimi.

\begin{abstract}
Surimi, a deboned, minced and washed fish muscle, is generally used as a raw material in the preparation of a variety of products, the consumption of which has increased over the years. During the preparation of surimi or surimi products, applied freezing or heating is sufficient to kill Anisakis larvae; however the presence of dead larvae can still cause allergic symptoms in sensitized consumers. The aim of this study was to evaluate the presence and viability of $A$. simplex larvae in the minced muscle and their retention in the sieves after pressing the infested muscle through individual (simulation of mincing) or successive sieves (simulation of mincing and refining) with different hole diameters. Fresh hake fillets (Merluccius merluccius) infested with $A$. simplex live larvae (20 larvae/100 g muscle) (controlled infestation) and a mixture of frozenthawed hake belly flaps with a heavy infestation (average 90 larvae/100 g of muscle) (natural infestation) were used and the presence of larvae in the fresh minced muscle and the retention of larvae in the sieves were measured. The yield of minced muscle obtained after sieving, ranged from 60 to $80 \%$ depending on the sieve used as well as the raw material. The percentage of entire larvae (alive or dead) in the minced muscle decreased significantly at lower hole's diametesr and after passing through successive sieves. The high content of broken larvae found might indicate a higher spread of antigens larvae in the sieved muscle.
\end{abstract}

Key words: Anisakis simplex, mechanical separation, surimi.

\section{Introducción.}

Surimi es un término japonés que significa músculo de pescado picado, lavado y con ingredientes adicionados para facilitar su conservación. Su proceso de elaboración implica eliminar espinas, tejido conectivo y todo aquello que puede considerarse no 
funcional, para conseguir una concentración de actomiosina con un contenido acuoso similar al original del músculo de pescado (Suzuki, 1981; Tejada, 1991; Park \& Lin, 2005). El surimi no es un producto final sino una materia prima, que por sus propiedades funcionales es utilizada para crear e imitar texturas y que puede servir de base para la elaboración de una amplia gama de productos, como el tradicional gel kamaboko japonés o productos de imitación de mariscos (Tejada \& Borderías, 1987; Carvajal et al., 2005; Blanco et al., 2006).

El procesamiento de surimi se puede dividir en dos etapas principales; la primera fase prepara el músculo de pescado, mientras que la segunda es la que implica el lavado y el tamizado del músculo picado (Suzuki, 1981; Martín-Sánchez et al., 2009). Durante la preparación del músculo y dependiendo del tamaño del pescado, la separación de las partes blandas (músculo) de las porciones más groseras (espinas, piel, aletas y escamas) puede realizarse en equipos que introducen el pescado entre una cinta móvil de material flexible y un tambor con orificios de 3-5 mm de diámetro y una presión que varía en función del músculo a tratar (Suzuki, 1981; Lee, 1984). La presión ejercida por la cinta; extrusiona el músculo a través de las perforaciones hacia el interior del tambor, logrando de esta forma una separación parcial del músculo ya que pequeñas espinas, algunas escamas y tejido conectivo también pueden pasar a través de los orificios de la criba (Tejada \& Borderías, 1987). Industrialmente se realiza una operación de refinado posterior a la etapa de lavados. En la refinadora un grupo de cuchillas, que giran a elevada velocidad, impulsan el músculo picado a través de pequeños orificios del tambor (1-2 mm) (Park \& Lin, 2005), saliendo el músculo finamente triturado al exterior, mientras que los elementos más groseros permanecen en el interior del equipo (Tejada \& Borderías, 1987). El rendimiento del músculo al final de esta etapa varía entre $20-50 \%$ dependiendo del tamaño y especie de pescado y del tamaño de los orificios del tambor (Shimizu \& Toyohara, 1992).

En la actualidad un alto porcentaje de pescado utilizado para el consumo humano se encuentra infestado de larvas de Anisákidos, sobre todo de Anisakis s.l. y Pseudoterranova sp. (Petrie et al., 2007; EFSA, 2010). La prevalencia y el grado de intensidad dependerán del caladero y de la especie de pescado entre otros factores. La presencia de larvas L3 de Anisakis en el pescado además de generar rechazo en el consumidor, ocasiona problemas sanitarios ya que al encontrarse vivas pueden producir infestación aguda o crónica y sus alérgenos causar alergia en los consumidores (Ward et al., 1997; Audicana et al., 2002; Audicana \& Kennedy, 2008). La legislación en la UE determina que los parásitos visibles tienen que ser eliminados y en el caso de que el número de parásitos sea alto, las piezas infestadas deben ser rechazadas, no permitiéndose su ingreso en la cadena alimentaria, lo que en algunos casos significa pérdidas altas para la industria pesquera. Sin embargo, no está especificado el número de larvas por unidad de peso o de pescado que daría lugar a su eliminación (Directiva 91/493/CEE; Reglamentos 853/2004; 854/2004; $1276 / 2011)$. En el surimi y productos elaborados con surimi, la congelación durante su conservación o el calentamiento durante el procesado de los geles son suficientes para matar las larvas de Anisakis (Tejada et al., 2006b; Rodriguez-Mahillo et al., 2010; Vidaček et al., 2009; 2010; 2011). Esto permitiría la utilización de pescado infestado en condiciones en las que se evitaría la infestación del consumidor y el rechazo a su adquisición, ya que debido al tratamiento de las muestras las larvas no son aparentes. Sin embargo, las larvas muertas todavía pueden causar síntomas de alergia en los consumidores previamente sensibilizados (Montoro et al., 1997; Audicana et al., 2002; Moneo et al., 2005; AAITO-IFIACI Anisakis Consortium, 2011).

El objetivo del estudio fue evaluar en la primera etapa del proceso general de obtención de surimi, la presencia y viabilidad de larvas de A. simplex en el músculo tamizado y su retención en los tamices después de pasar el músculo infestado a través de tamices individuales (simulación de extrusión) o sucesivos (simulación de extrusión y refinado) con diferentes diámetros de orificio.

\section{Materiales y métodos.}

\section{Larvas de Anisakis spp.}

Las larvas vivas de Anisakis en la tercera fase (L3) fueron obtenidas de ovarios de merluzas (Merluccius merluccius) infestadas, procedentes del Atlántico noroeste, recepcionadas en el Mercado Central de Pescado de Madrid (Mercamadrid) en el mes de marzo del 2011 y enviadas a las instalaciones del Instituto de Ciencia y Tecnología de Alimentos y Nutrición (ICTAN) en Madrid, España. Se utilizó un lote de aproximadamente $100 \mathrm{~g}$ de larvas incluyendo tejidos de ovarios que se almacenaron hasta su utilización a $5 \pm 1^{\circ} \mathrm{C}$ durante un tiempo máximo de 24 horas.

\section{Pescado utilizado}

Para el estudio se utilizaron merluzas (Merluccius merluccius) capturadas en la zona de Pesca FAO 27 en junio del 2010, seleccionadas en Mercamadrid y posteriormente enviadas al ICTAN. La longitud y el peso de los individuos fue $44.25 \pm 1.32 \mathrm{~cm}$ y $0.92 \pm 0.02$ $\mathrm{kg}$ respectivamente. Las 4 merluzas recepcionadas fueron descabezadas, evisceradas, envasadas al vacío $\left(10.66 \times 10^{3} \mathrm{~Pa}\right)$ en bolsas plásticas (Cryovac BB-1, Duncan, SC, USA; permeabilidad al oxígeno a $23^{\circ} \mathrm{C}$, $60 \mathrm{~cm}^{3} /$ día-m ${ }^{2}$-atm) y conservadas en congelación $\left(-20 \pm 2^{\circ} \mathrm{C}\right)$ durante 9 meses. Las merluzas fueron descongeladas y cortadas en filetes, utilizando los filetes dorsales sin piel $(2.01 \pm 0.05 \mathrm{~kg})$ para el estudio 
de infestación controlada (lote 1) y los músculos ventrales para el estudio de infestación natural (lote 2). La temperatura durante la manipulación de las muestras fue $\leq 5^{\circ} \mathrm{C}$.

Para el lote 2, debido a la baja tasa de infestación ( $\leq 5$ larvas por $100 \mathrm{~g}$ de músculo) del músculo ventral inicial, se utilizó un lote adicional de músculos ventrales con alta tasa de infestación (116 larvas por $100 \mathrm{~g}$ de músculo) obtenidos de merluzas capturadas en la zona de pesca VIa-FAO 27, envasadas al vacío en bolsas plásticas y conservadas en congelación a $30 \pm 3^{\circ} \mathrm{C}$ durante 16 meses. La determinación de la infestación se realizó contabilizando la cantidad de larvas enteras luego de digestión con pepsina (Ozanz Mur, 2001) de los músculos ventrales y calculando la media. En total se utilizó una mezcla de $2.00 \pm 0.05 \mathrm{~kg}$ de músculos ventrales (izquierdos y derechos) previamente descongelados en cámara a $\leq 5^{\circ} \mathrm{C}$ y quitada la piel.

Preparación de la muestra

Dependiendo del tipo de músculo a utilizar se prepararon 2 lotes, que se estudiaron individualmente. En el lote 1 (infestación controlada) las larvas vivas de Anisakis, se colocaron entre dos capas de músculo dorsal (tipo sándwich) en una proporción de 20 larvas por $100 \mathrm{~g}$ de músculo. Los sándwiches infestados se almacenaron en refrigeración $\left(5 \pm 1^{\circ} \mathrm{C}\right)$ durante 48 horas para favorecer la penetración de las larvas en el músculo (Tejada et al., 2006b).

Para el lote 2 (infestación natural) se utilizó una mezcla de músculos ventrales con diferente tasa de infestación natural, tal como se describe previamente. La tasa final de infestación se obtuvo mezclando músculo parasitado de una tasa estimada de 116 larvas con músculo ventral de las merluzas utilizadas para parasitación controlada, aparentemente libre de larvas, por lo que la tasa final promedio se estimó en 90 larvas por $100 \mathrm{~g}$ de músculo.

En cada lote, los músculos infestados fueron troceados con cuchillo y homogenizados en una batidora-picadora (Hand processor accesory, Minipimer 5, Braun $\mathrm{GmbH}$, Alemania) a baja velocidad y con toques suaves a fin de evitar romper las larvas. El homogenizado obtenido para cada lote fue distribuido en 18 muestras de $100 \mathrm{~g}$ cada una. La operación de tamizado (simulación de extrusión y refinado) se realizó con un molino de alimentos (OXO Good Grips, UK) utilizando tamices de diferentes diámetros de orificio (Tamiz A: $\varnothing=3.5 \mathrm{~mm}$; Tamiz B: $\varnothing=3.0 \mathrm{~mm}$; Tamiz C: $\varnothing=2.0 \mathrm{~mm})$. Ambos lotes fueron pasados por separado a través de tamices individuales o sucesivos. Para cada lote se obtuvieron 6 tipos de músculos tamizados (fracciones $\mathrm{MTn}_{\mathrm{x}}$ ) con sus correspondientes residuos retenidos en el tamiz (fracciones $\mathrm{RTn}_{\mathrm{x}}$ ) y cada operación de tamizado se realizó por triplicado. La temperatura de las muestras durante el procesado fue $\leq 5^{\circ} \mathrm{C}$.

\section{Métodos}

Identificación taxonómica de larvas de Anisakis spp.

Para los estudios de identificación taxonómica, las larvas recepcionadas fueron separadas cuidadosamente del tejido (ovarios) con pinzas y sometidas a lavados sucesivos con agua destilada. Las larvas limpias, se congelaron $\left(-30 \pm 3^{\circ} \mathrm{C}\right)$ y se enviaron al Museo Nacional de Ciencias Naturales (MNCN) para su estudio. La extracción de ADN fue realizada en el MNCN de acuerdo al protocolo descrito por Holmes y Bonner (1973) y modificado por D'Amelio et al. (2000).

Análisis elementales y $\mathrm{pH}$

Como control de la muestra de merluzas se determinó el contenido de humedad, cenizas (AOAC, 2005), proteína bruta [Analizador de nitrógeno/proteína LECO FP-2000 (LECO Corp., St Joseph, MI, EE.UU), utilizando un factor de conversión de nitrógeno a proteína de 6.25] y grasa bruta (Smedes, 1999; Karl et al., 2012) en el músculo inicial. Los análisis se realizaron al menos por triplicado y los resultados se expresaron en $\mathrm{g} \mathrm{kg}^{-1}$. El $\mathrm{pH}$ se determinó a temperatura ambiente usando $10 \mathrm{~g}$ de músculo picado en $100 \mathrm{ml}$ de agua destilada. Las medidas se realizaron con un electrodo de vidrio (electrodo de pH línea azul Elektrolyt L300; Schott Instruments, Mainz, Alemania).

\section{Cuantificación y viabilidad de las larvas}

\section{Digestión con pepsina}

Para cuantificar las larvas las fracciones $\mathrm{MTn}_{\mathrm{x}} \mathrm{y}$ $\mathrm{RTn}_{\mathrm{x}}$ fueron digeridas con solución de pepsina a una concentración final de $0.3 \mathrm{M} \mathrm{HCl}, 10 \mathrm{mg} \mathrm{ml}^{-1}$ pepsina [actividad proteolítica 1:10000 NF (2000 FIP-U g-1), Panreac, Castellar del Vallés, España] (1:2; p:v), $\mathrm{pH} \leq 1.1$ (CODEX, 2004). Se incubaron en baño de agua $\left(37 \pm 0.5^{\circ} \mathrm{C}\right)$ con agitación constante durante 30 minutos o hasta que el músculo estuvo completamente digerido (Ozanz Mur, 2001; Vidaček et al., 2009). Una vez terminada la digestión, se enfriaron inmediatamente en agua con hielo y se neutralizaron para evitar que la pepsina continuara ejerciendo su efecto. Para facilitar la cuantificación de las larvas enteras, las fracciones digeridas de ambos lotes fueron filtradas a vacío en matraces kitasato y filtros de papel Whatman \#1 (Cat N ${ }^{\circ} 1001$ 125, GE Healthcare UK Limited), evaluando la parte retenida en los filtros de cada fracción $\left(\mathrm{MTn}_{\mathrm{x}} \mathrm{y} \mathrm{RTn}_{\mathrm{x}}\right)$.

\section{Movilidad}

Este procedimiento sólo se realizó en las fracciones $\operatorname{MTn}_{\mathrm{x}}$ y $\mathrm{RTn}_{\mathrm{x}}$ del lote 1 (infestación controlada con larvas vivas). Se verificó visualmente el movimiento espontáneo o estimulado (tocando las larvas con una aguja sin dañar la larva) de las larvas encontradas después de filtrar al vacío las fracciones digeridas. Cuando no se observó movimiento durante un periodo de 2 horas se consideró que la larva estaba muerta (Solas et al., 2008; Vidaček et al., 2010). Se 
contabilizaron las larvas vivas encontradas en cada fracción.

\section{Emisión de fluorescencia}

Para verificar la presencia, integridad y cuantificar las larvas en cada fracción $\left(\mathrm{MTn}_{\mathrm{x}} \mathrm{y} \mathrm{RTn}_{\mathrm{x}}\right)$ y lote $(1 \mathrm{y}$ 2), se evaluó la emisión de fluorescencia (366 nm) del material retenido en los filtros después de congelarlos a $-20 \pm 2^{\circ} \mathrm{C}$ durante 48 horas (Tejada et al., 2006a). Para facilitar el recuento se tomaron fotografías con una cámara digital (Coolpix 5000, Nikon, Tokyo, Japón) con luz visible y luz ultravioleta (366 nm). En los filtrados de todas las fracciones $\left(\mathrm{MTn}_{\mathrm{x}} \mathrm{y} R \mathrm{RT}_{\mathrm{x}}\right)$ y lotes ( 1 y 2) se contabilizaron las larvas enteras y por diferencia se obtuvo la cantidad de larvas rotas. El resultado se calculó en relación con el porcentaje de larvas presentes inicialmente en el músculo.

Microscopia electrónica de barrido (SEM)

El análisis sólo se realizó en las muestras correspondientes al lote 2 (infestación natural, larvas muertas) y tuvo como finalidad evaluar las modificaciones en el músculo tamizado. Se aplicó el protocolo descrito por Tejada et al. (2006a).

Resultados y discusión.

Identificación taxonómica de larvas de Anisakis

Morfológicamente las larvas del lote 1 fueron clasificadas como tipo I e identificadas como Anisakis simplex s.s. (88.57\%) y Anisakis pegreffii (11.42\%).

Análisis elementales y $\mathrm{pH}$

El contenido de humedad $\left(805.36 \pm 2.31 \mathrm{~g} \mathrm{~kg}^{-1}\right)$, cenizas $\left(13.08 \pm 0.08 \mathrm{~g} \mathrm{~kg}^{-1}\right)$, proteína $(191.17 \pm 1.30 \mathrm{~g}$ $\left.\mathrm{kg}^{-1}\right)$ y grasa $\left(8.15 \pm 0.10 \mathrm{~g} \mathrm{~kg}^{-1}\right)$ del músculo de merluza se encuentra dentro de los valores reportados para la especie (Tejada et al., 2003).

$\underline{\text { Rendimiento del músculo }}$

El rendimiento del músculo tamizado respecto al músculo inicial osciló entre 60 y $80 \%$, dependiendo del tamiz utilizado y del lote estudiado (Figura 1). No se encontraron diferencias significativas en el rendimiento entre los lotes 1 y 2 para el mismo tipo de tamiz. Sin embargo, se aprecia en el mismo lote una disminución significativa en el rendimiento cuando el músculo pasa a través de tamices sucesivos y se obtuvieron los menores rendimientos cuando las muestras se sometieron al tamizado con tres tamices sucesivos $(\mathrm{ABC})$, encontrándose en este caso diferencias significativas entre lotes $y$ con un rendimiento menor en el lote 1 compuesto por músculo dorsal.

Cuantificación de las larvas

En la Figura 2 se muestra el porcentaje de larvas enteras en el músculo tamizado $\left(\mathrm{MTn}_{\mathrm{x}}\right)$ y retenidas en los distintos tamices $\left(\mathrm{RTn}_{\mathrm{x}}\right)$. En todos los casos se aprecia una disminución del número de larvas enteras al disminuir el tamaño de los orificios del tamiz o incrementar el paso a través de distintos tamices, lo que significa que hay una rotura mayor de las larvas en estas condiciones. No obstante en el lote 2, excepto en el tamiz A $(\varnothing=3.5 \mathrm{~mm})$, se aprecia un mayor porcentaje de larvas enteras en ambas fracciones, probablemente debido a que las modificaciones que sufre la cutícula de las larvas en congelación las hace más resistentes a la rotura. En ambos lotes, el efecto fue mayor en el músculo tamizado $\left(\mathrm{MTn}_{\mathrm{x}}\right)$ que en la fracción retenida en el tamiz $\left(\mathrm{RTn}_{\mathrm{x}}\right)$. Las larvas rotas que pasan al músculo, pueden producir una mayor propagación de antígenos en el músculo, sobre todo después de utilizar tamices con un orificio de diámetro más pequeño. Hay que tener en cuenta que la presión ejercida en el proceso de extrusionado a través de los distintos tamices puede modificar los porcentajes obtenidos en las distintas fracciones cuando se utilizan otras condiciones de procesado.

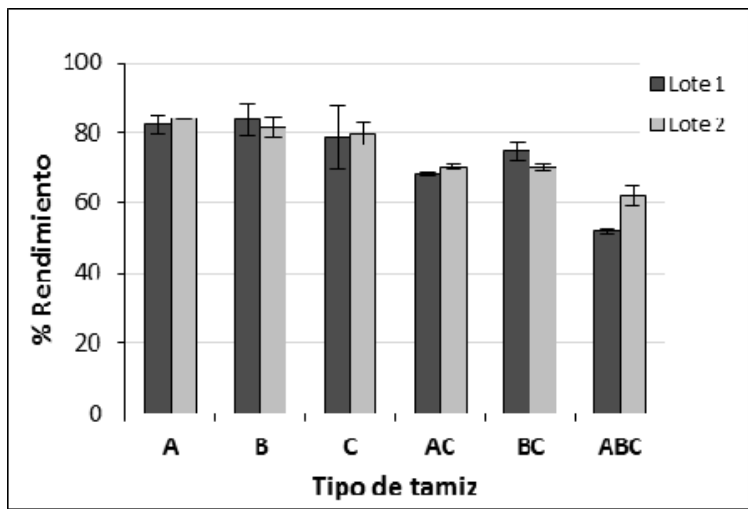

Figura 1. Rendimiento (\%) del músculo de merluza obtenido después de la operación de tamizado. Lote 1: Músculo dorsal con infestación controlada (larvas vivas, 20 larvas/100 g músculo); Lote 2: Músculo ventral con infestación natural (larvas muertas, $\cong 90$ larvas/100 g músculo). Diámetro de los orificios de los tamices: Tamiz A=3.5 mm; Tamiz $B=3.0 \mathrm{~mm}$; Tamiz $\mathrm{C}=2.0 \mathrm{~mm}$.

\section{$\underline{\text { Viabilidad de las larvas }}$}

Cuando se utilizó el tamiz A se detectó el 20\% de larvas vivas sobre las inicialmente presentes en el músculo y el $5 \%$ con las del tamiz $\mathrm{B}$, confirmando la resistencia de las larvas en condiciones de acidez extrema. Sin embargo, se debe considerar que las larvas con cutícula intacta se mantienen vivas y enteras en condiciones de digestión, mientras que si la cutícula se encuentra alterada por algún proceso, las larvas se pueden digerir y su recuento sería menor del esperado (Rodríguez-Mahillo et al., 2008; Solas et al., 2009).

En la industria una operación de refinado, dependiendo del diámetro de orificio del tambor, podría significar una menor cantidad de larvas en el músculo utilizado posteriormente para la elaboración de surimi, considerándose que este proceso disminuye la presencia de larvas en lotes de pescado o zonas anatómicas con alta tasa de infestación. Sin embargo, existe la posibilidad de que una mayor rotura de las 
larvas al disminuir el diámetro de los orificios pueda influir en la dispersión de alérgenos en el músculo.

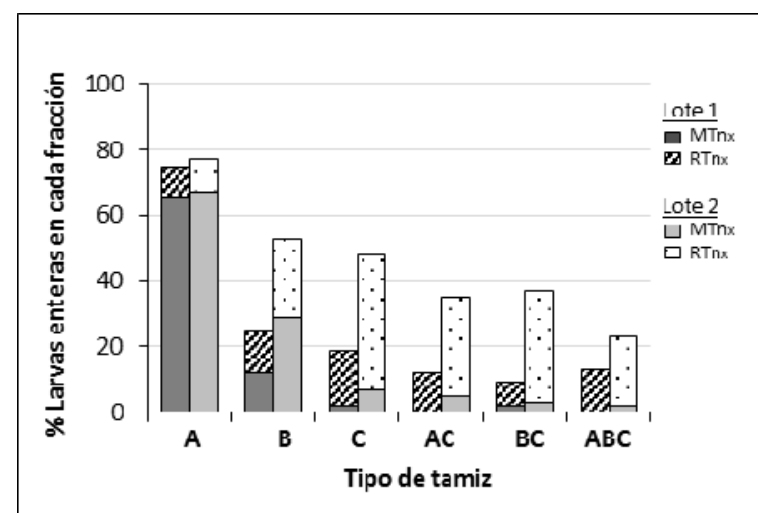

Figura 2. Porcentaje (\%) de larvas L3 de Anisakis enteras en músculo tamizado de merluza $\left(\mathrm{MTn}_{\mathrm{x}}\right)$ y en los residuos retenidos en el tamiz $\left(\operatorname{RTn}_{\mathrm{x}}\right)$. Lote 1 : Músculo dorsal con infestación controlada (larvas vivas, 20 larvas/100 g músculo); Lote 2: Músculo ventral con infestación natural (larvas muertas, $\cong 90$ larvas/100 g músculo); Diámetro de los orificios de los tamices: Tamiz $\mathrm{A}=3.5 \mathrm{~mm}$; Tamiz $\mathrm{B}=3.0 \mathrm{~mm}$; Tamiz $\mathrm{C}=2.0 \mathrm{~mm}$.

\section{Microestructura del músculo tamizado}

En la Figura 3 se presentan imágenes de microscopia electrónica de barrido (SEM) del músculo ventral tamizado $\left(\mathrm{MTn}_{\mathrm{x}}\right.$, lote 2). El estudio microscópico del músculo nos indicó que existe un proceso gradual de ruptura de las fibras musculares por la acción del tamizado. Las diferencias estructurales en el músculo se observaron mejor en las muestras que fueron refinadas con tamices sucesivos, en especial aquellas con menor diámetro de orificio. Sin embargo, incluso en la muestra obtenida con la combinación de tamices $\mathrm{ABC}$, se encontró la presencia de larvas prácticamente enteras (Figura 4). El diámetro de las larvas L3 oscila entre 0.10 y 0.15 $\mathrm{mm}$ lo que justificaría su presencia en el músculo tamizado y la mayor cantidad de larvas enteras encontradas cuando se utilizó el tamiz A.

\section{Conclusiones.}

1. La operación de tamizado durante el proceso de elaboración de surimi ocasionó la retención de larvas enteras en el tamiz, que estuvo inversamente relacionada con el diámetro de los orificios, e implicó una disminución en la cantidad de larvas enteras en el músculo tamizado correspondiente. Actualmente se están realizando estudios inmunológicos con el fin de dilucidar cómo esta primera fase del proceso influye en la tasa de antígenos en el surimi obtenido.
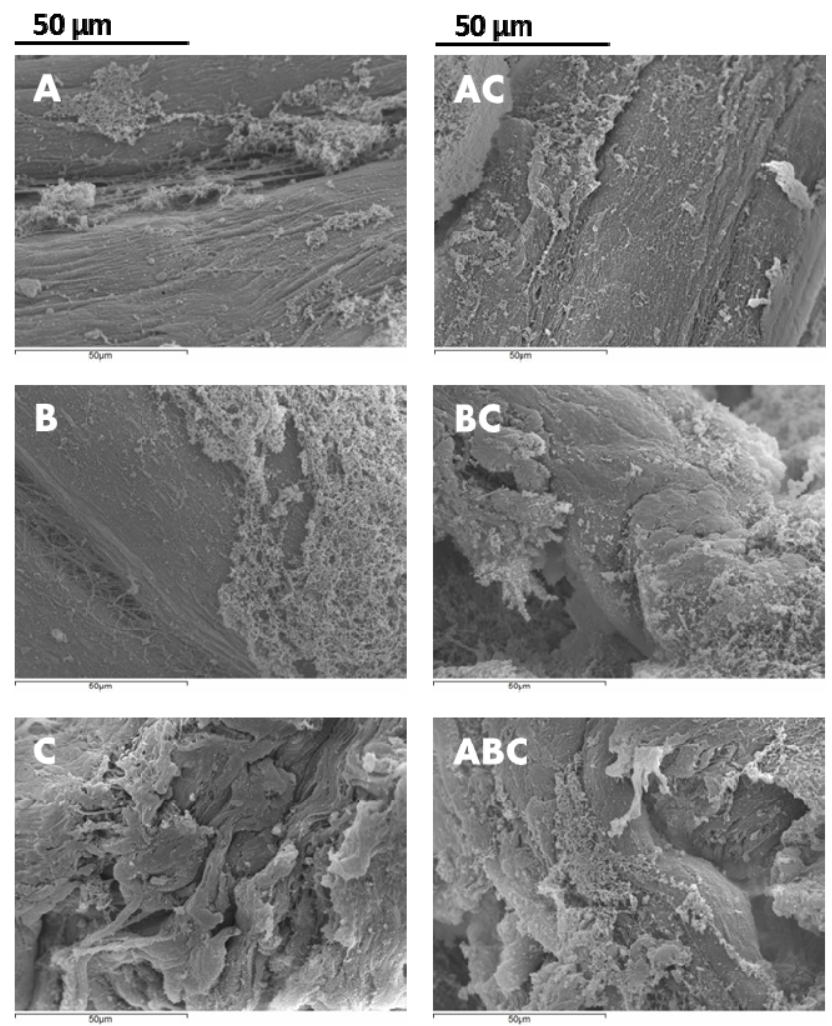

Figura 3. Microscopia electrónica de barrido (SEM) de músculo ventral de merluza con infestación natural (lote 2) obtenido después de la operación de tamizado. Tipo de tamizado de acuerdo al diámetro de los orificios de los tamices: A-Tamiz A $(\varnothing=3.5 \mathrm{~mm})$; B-Tamiz B $(\varnothing=3.0$ $\mathrm{mm})$; C-Tamiz C $(\varnothing=2.0 \mathrm{~mm})$; AC-Combinación de tamices A y C; BC-Combinación de tamices $\mathrm{B}$ y C; $\mathrm{ABC}$ Combinación de tamices A, B y C.

\section{Agradecimientos.}

El presente trabajo ha sido realizado en el ICTANCSIC de Madrid y se ha financiado por el proyecto del Plan Nacional I+D+i español AGL2009-12485-C0301/03 (ANIDET). Fabiola Olivares ha realizado el trabajo en el ICTAN mediante una beca de estudios concedida por el Programa de Ciencia y Tecnología del Gobierno de Perú (FINCyT) administrada por LASPAU. Agradecemos al Sr. Angel Mendizábal de Madrid-Salud (Ayuntamiento de Madrid), Instituto de Salud Pública-Seguridad Alimentaria (Unidad Técnica Mercamadrid) Madrid, España, por el suministro de larvas de A. simplex; y al Dr. Alfonso Navas del Museo Nacional de Ciencias Naturales (MNCNCSIC) por la identificación de las larvas utilizadas en el presente trabajo. 


\section{$200 \mu \mathrm{m}$}

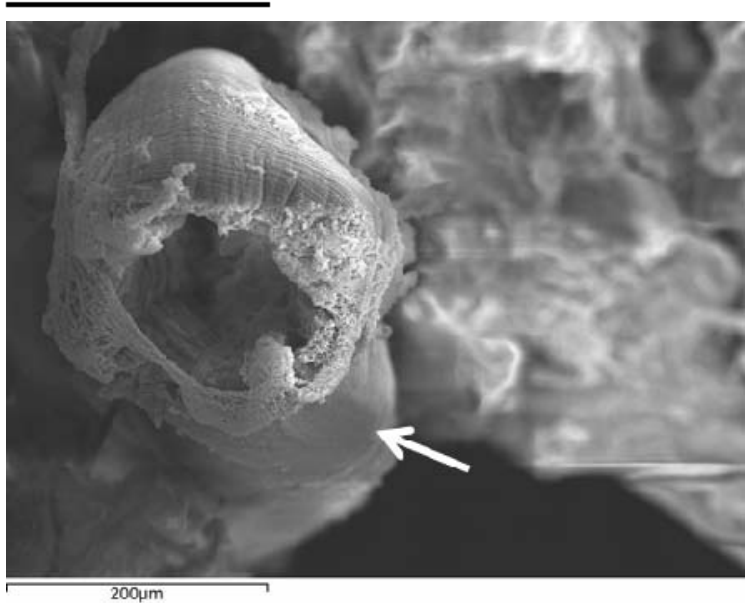

Figura 4. Micrografía del cuerpo de una larva de Anisakis spp. encontrada en músculo ventral de merluza (lote 2) tamizado con una combinación de tamices sucesivos (ABC). La presencia de la larva se indica con una flecha.

\section{Literatura citada.}

AAITO-IFIACI Anisakis Consortium. 2011. Anisakis hypersensitivity in Italy: prevalence and clinical features: a multicenter study. Allergy 66: 1563-1569.

AOAC. 2005. Official Methods of Analysis, 18th ed. Association of Official Analytical Chemists, Arlington, VA, USA.

Audicana M. \& Kennedy M. 2008. Anisakis simplex: from Obscure Infectious Worm to Inducer of Immune Hypersensitivity. Clin. Microbiol. Rev., 21(2): 360-379.

Audicana M., Ansotegui I., Fernández de Corres L. \& Kennedy M. 2002. Anisakis simplex: dangerous-dead and alive?. Trends Parasitol., 18: 20-25.

Blanco M., Sotelo C., Chapela M. \& Pérez-Martín R. 2006. Towards sustainable and efficient use of fishery resources: present and future trends. Trends Food Sci. Technol., 18(1): 29-36.

Carvajal P., Lanier T. \& Mac Donald G. 2005. Stabilization of proteins in surimi. En: Park J (ed) Surimi and surimi seafood: 163-225. Taylor \& Francis Group, Boca Ratón, Florida.

CODEX. 2004. Standard for salted atlantic herring and salted sprat. CODEX STAN 244-2004.

D'Amelio S., Mathiopoulos K.D., Santos C.P., Pugachev O.N., Webb S.C. Picanço M. \& Paggi L. 2000. Genetic markers in ribosomal DNA for the identification of members of the genus Anisakis (Nematoda: Ascaridoidea) defined by polymerase chain reaction based restriction fragment length polymorphism. Int. J. Parasitol., 30: 223-226.

Directiva 91/493/CEE del Consejo de 22 de Julio de 1991 por la que se fijan las normas sanitarias aplicables a la producción y a la puesta en el mercado de los productos pesqueros. Diario Oficial de las Comunidades Europeas, DOCE L268/15 del 24.09.91. Disponible en: http://eurlex.europa.eu/
LexUriServ/LexUriServ.do?uri=OJ:L:1991:268:0015:00 34:ES:PDF

EFSA. 2010. Scientific Opinion on risk assessment of parasites in fishery products. European Food Safety Authority (EFSA) Panel on Biological Hazards (BIOHAZ). EFSA Journal, 8(4): 1543.

Karl H., Bekaert K., Bergé J., Cadun A., Duflos G., Tejada M., Testi S. \& Timm-Heinrich M. 2012. WEFTA Interlaboratory comparison on total lipid determination in fishery products using the Smedes Method. J. AOAC Int., 95(2): 489-493.

Lee C. 1984. Surimi process techonology. Food Technol., 38(11): 69-80.

Martín-Sánchez A., Navarro C., Pérez-Alvarez J. \& Kuri V. 2009 Alternatives for efficient and sustainable production of surimi: A Review. Comprehensive Reviews in Food Science and Food Safety, 8: 359-374.

Moneo I., Caballero M., González-Muñoz M., RodriguezMahillo A., Rodriguez-Pérez R. \& Silva A. 2005. Isolation of a heat resistant allergen from the fish parasite Anisakis simplex. Parasitol. Res., 96: 285-289.

Montoro A., Perteguer M., Chivato I., Laguna R. \& Cuellar C. 1997. Recidivous acute urticaria caused by Anisakis simplex. Allergy, 52: 985-991.

Osanz Mur A. 2001. Presencia de larvas de Anisákidos (Nematoda: Ascaroidea) en pescado de consumo capturado en la zona pesquera de Tarragona. Tesis Doctoral. Universidad Autónoma de Barcelona. Facultad de Veterinaria.

Park J. \& Lin J. 2005. Surimi: Manufacturing and evaluation. En: Park J (ed) Surimi and surimi seafood: 33-106. Taylor \& Francis Group, Boca Ratón, Florida.

Petrie A., Wootten R., Bruno D., MacKenzie K. \& Bron J. 2007. A survey of Anisakis and Pseudoterranova in Scottish fisheries and the efficacy of current detection methods. FSAS Project S14008.

Reglamento 1276/2011 de la comisión de 8 de diciembre de 2011 que modifica el anexo III del Reglamento 853/2004 del Parlamento Europeo y del Consejo en lo referente al tratamiento para matar parásitos viables en los productos de la pesca destinados al consumo humano. Diario Oficial de la Unión Europea, DOUE L327/39 de 9.12.2011. Disponible en: http://eurlex.europa.eu/

LexUriServ/LexUriServ.do?uri=OJ:L:2011:327:0039:00 41:ES:PDF

Reglamento 853/2004 del Parlamento Europeo y del Consejo de 29 de abril de 2004 por el que se establecen normas específicas de higiene de los alimentos de origen animal. Diario Oficial de la Unión Europea, DOUE L139/55 de 30.04.2004. Disponible en: http://eurlex.europa.eu/LexUriServ/Lex UriServ.do?uri=OJ:L:2004:139:0055:0205:ES:PDF

Reglamento 854/2004 del Parlamento Europeo y del Consejo de 29 de abril de 2004 por el que se establecen normas específicas para la organización de controles oficiales de los productos de origen animal destinados al consumo humano Diario Oficial de la Unión Europea, DOUE L139/206 de 30.4.2004. Disponible en: http://eur-lex.europa.eu/LexUriServ/LexUriServ.do? uri=OJ:L:2004:139:0206:0320:ES:PDF

Rodríguez-Mahillo A., González-Muñoz M., De las Heras C., Tejada M. \& Moneo I. 2010. Quantification of Anisakis simplex allergens in fresh, long-term frozen and 
cooked fish muscle. Foodborne Pathog. Dis., 7(8): 967973.

Rodríguez-Mahillo A., González-Muñoz M., Moneo I., Solas M., Mendizábal A., De las Heras C. \& Tejada M. 2008. Allergenic properties and cuticle microstructure of Anisakis simplex L3 after freezing and pepsin digestion. J. Food Protect., 71(12): 2578-2581.

Shimizu Y. \& Toyohara H. 1992. Fish jelly products. JICA, Japan International Cooperation Agency.

Smedes F. 1999. Determination of total lipid using nonchlorinated solvents. Analyst, 124: 1711-1718.

Solas M., García M., De las Heras C., Rodríguez-Mahillo A., González-Muñoz M. Moneo I., Mendizábal A. \& Tejada M. 2009. Anisakis simplex antigens in fresh and frozen-thawed muscle of anchovies in vinegar. Food Sc. Tech. Int., 15(2): 139-148.

Solas M., García M., Rodriguez-Mahillo A., GonzálezMuñoz A., De las Heras C. \& Tejada M. 2008. Anisakis antigens detected in fish muscle infested with Anisakis simplex L3. J. Food Protect., 71(6): 1273-1276.

Suzuki T. 1981. Fish and Krill Protein: Processing Technology. Applied Science Publ., Ltd,. Essex, UK.

Tejada M. \& Borderías J. 1987. Productos derivados del surimi. Rev. Agroquím. Tecnol. Aliment., 27(2): 161172.

Tejada M. 1991. Tendencias actuales en la utilización de surimi. Rev. Agroquím. Tecnol. Aliment., 31(3): 310318.

Tejada M., Mohamed G., Huidobro A. \& García M. 2003. Effect of frozen storage of hake, sardine and mixed minces on natural actomyosin extracted in salt solutions. J. Sci. Food Agric., 83: 1380-1388.
Tejada M., Solas, M., Navas A. \& Mendizábal A. 2006 a. Scanning electron microscopy of Anisakis larvae following different treatments. J. Food Protect. 69(6): 1379-1387.

Tejada M., Solas M., Navas A. \& Mendizábal A. 2006b. Effect of freezing and different heat treatments on Anisakis larvae: preliminary study. En: J.B. Luten, C. Jacobsen, K. Bekaert, A. Sæbø, J. Oehlenschläger (eds) Seafood research from fish to dish. Quality, safety and processing of wild and farmed fish: 309-316. Wageningen Academic Publishers.

Vidaček S., De las Heras C., Solas M., García M., Mendizábal A. \& Tejada M. 2011. Viability and antigenicity of Anisakis simplex after conventional and microwave heating at fixed temperatures. J. Food Protect. 74 (12): 2119-2126.

Vidaček S., De las Heras C., Solas M., Mendizabal A., Rodriguez-Mahillo A., González-Muñoz M. \& Tejada M. 2009. Anisakis simplex allergens remain active after conventional or microwave heating and pepsin treatments of chilled and frozen L3 larvae. J. Sci. Food Agric., 89: 1997-2002.

Vidaček S., De las Heras C., Solas M., Mendizábal A., Rodríguez-Mahillo A. \& Tejada M. 2010. Antigenicity and Viability of Anisakis larvae heated at different timetemperature conditions. J. Food Protect., 73(1): 62-68.

Ward D., Bernard D., Collette R., Kraemer D., Hart K., Price R. \& Otwell S. 1997. Hazards Found in Seafoods, Appendix III. En: HACCP: Hazard Analysis and Critical Control Point Training Curriculum: 173-188. UNC-SG96-02; North Carolina Sea Grant, Raleigh, NC.

\footnotetext{
${ }^{1}$ Facultad de Pesquería, Universidad Nacional Agraria La Molina (UNALM), Av. La Molina s/n, La Molina, Lima, Perú. E-mail: folivares@lamolina.edu.pe

${ }^{2}$ Instituto de Ciencia y Tecnología de Alimentos y Nutrición (ICTAN), Consejo Superior de Investigaciones Científicas (CSIC), C/José Antonio Novais 10, 28040 Madrid, España. E-mail: cha@ictan.csic.es

${ }^{3}$ Departamento de Biología Celular, Facultad de Ciencias Biológicas, Universidad Complutense de Madrid (UCM), C/José Antonio Novais 2, 28040 Madrid, España. E-mail: msolas@bio.ucm.es

${ }^{4}$ Instituto de Ciencia y Tecnología de Alimentos y Nutrición (ICTAN), Consejo Superior de Investigaciones Científicas (CSIC), C/José Antonio Novais 10, 28040 Madrid, España. E-mail: mtejada@ictan.csic.es
} 